\title{
Qualidade de vida do cuidador durante internação da pessoa cuidada em Unidade de Urgência/Emergência: alguns fatores associados
}

\author{
Quality of life of caregivers during the hospitalization of the patient \\ under care in an Emergency Unit: some associated factors
}

Tânia Madureira Dallalana ${ }^{1}$

Maria Geny Ribas Batista ${ }^{1}$

\footnotetext{
${ }^{1}$ Serviço de Psicologia, Hospital de Clínicas da Universidade Federal do Paraná. R. Gen. Carneiro 181, Alto da Glória. 80060-900 Curitiba PR Brasil.t.dalla@uol.com.br
}

\begin{abstract}
The purpose of this quantitative-descriptive study was to assess the quality of life of caregivers of patients hospitalized in emergency units of Hospital de Clinicas of the Federal University of Paraná. Tasks carried out by caregivers are significantly stressful and have a direct impact on their quality of life. From May to October 2011, 60 caregivers answered the WHOQOL-100 questionnaire developed by the World Health Organization. The majority of the interviewees were women $(n=47)$, with an average age of 43.2 years. Quality of life in general obtained an average score of 13.8. Results show that the quality of life of caregivers is vulnerable, which may affect their productivity. A sense of religiousness and spirituality, associated with a social-affective network are important resources to help them cope with the issues at hand.
\end{abstract}

Key words Caregiver, Quality of life, Urgency and emergency
Resumo Este estudo, de caráter quantitativodescritivo, teve como objetivo avaliar a qualidade de vida de cuidadores dos pacientes internados em unidades de urgência e emergência do Hospital de Clínicas da Universidade Federal do Paraná. As tarefas desenvolvidas pelo cuidador constituem eventos estressores significativos, afetando diretamente sua qualidade de vida. Foi aplicado a 60 cuidadores, de maio a outubro de 2011, o instrumento de avaliação WHOQOL-100, elaborado pela Organização Mundial da Saúde. Do total de participantes, predominou o sexo feminino $(n=$ 47), com idade média de 43,2 anos. A qualidade de vida geral obteve o escore médio de 13,8. Os resultados indicam que a qualidade de vida do cuidador se apresenta vulnerável, o que pode afetar a sua fase produtiva. A religiosidade, a espiritualidade e a ajuda da rede socioafetiva são importantes recursos de enfrentamento.

Palavras-chave Cuidador, Qualidade de vida, Urgência e emergência 


\section{Introdução}

O conceito de família exige uma reflexão comple$\mathrm{xa}$, que leve em conta a história afetiva e sociocultural individual e intergeracional de um homem e uma mulher no casamento, com funções e papéis determinados durante a passagem do ciclo vital familiar, em busca do sonho do futuro regado pelos afetos do passado.

Qualquer agravo de saúde que aconteça com um dos membros pode causar um processo de desorganização na psicodinâmica familiar. Segundo Martins et al. ${ }^{1}$, quando uma doença crônica e grave atinge um membro da família e acarreta internação hospitalar, todo o sistema familiar é atingido.

Saber quem é o cuidador principal, como e por que ele surge no sistema familiar e como a doença afeta seu estilo de vida é uma informação importante para o desenvolvimento de ações de colaboração da família com as equipes de saúde nos diferentes contextos no campo da saúde.

O impacto da doença na organização familiar é multidimensional, provocando alterações de ordem afetiva, financeira, social, cultural e espiritual, e trazendo consequências na adaptação das pessoas envolvidas com o cuidado, no tempo curto e longo da vida, no dia a dia. Por isso a importância de avaliar a qualidade de vida do cuidador na interface com o ato de cuidar.

As preocupações com a pessoa internada são constantes, acompanhadas de medo, angústia e insegurança. As alterações na rotina podem fazer com que os membros da família, principalmente o cuidador principal, sintam-se vulneráveis diante do sofrimento do outro e do papel que precisam assumir durante a internação e após a alta hospitalar ${ }^{2}$.

O presente estudo avaliou como o impacto subjetivo de uma doença crônica na família pode afetar a qualidade de vida do cuidador. Para compreender tal impacto, foi aplicado o Questionário de Avaliação de Qualidade de Vida, o WHOQOL-100 $0^{3}$ (OMS, 1997). A hipótese levantada é que o convívio com pacientes e as demandas do ato de cuidar experimentados pelas famílias e, em particular, pelo cuidador primário, podem causar uma alteração bastante significativa em sua dinâmica cotidiana e, consequentemente, na sua qualidade de vida. A trajetória teórica e conceitual desta pesquisa tem como base o paradigma pós-moderno e sua leitura feita por Edgar Morin $^{4}$, Humberto Maturana ${ }^{5}$, Bateson ${ }^{6}$, Bronfenbrenner ${ }^{7}$, Minayo ${ }^{8}$ e Spink ${ }^{9}$. Para esses autores, a relação do observador com o observado na ação da pesquisa implica a construção conjunta de conhecimento nos diferentes contextos.

\section{Métodos}

O método deste estudo é descritivo e transversal, com abordagem quantitativa. Em relação à idade, os participantes são maiores de 18 anos e se apresentam como cuidadores informais, ou seja, membros da família que estavam auxiliando nos cuidados ao paciente durante sua internação. Esse grupo falou sobre a sua qualidade de vida e não sobre a qualidade de vida dos familiares enfermos.

$\mathrm{O}$ instrumento utilizado foi o WHOQOL- $100^{3}$, um questionário cuja forma de coleta de dados consiste em questões pré-elaboradas referentes ao tema da pesquisa. É um instrumento de avaliação de qualidade de vida composto por 100 itens, baseado em seis domínios: domínio físico, domínio psicológico, nível de independência, relações sociais, meio ambiente e espiritualidade. O questionário foi respondido em um só encontro e com base nas duas últimas semanas de vida do cuidador. A escolha desse questionário deve-se ao fato de ele ser abrangente em relação aos aspectos da qualidade de vida, segundo Fleck et al..$^{10}$, porque mostra características psicométricas satisfatórias na população brasileira, além de incluir o aspecto espiritualidade.

De acordo com Fleck et al. ${ }^{11}$, após a elaboração desse questionário, surgiu a necessidade de instrumentos de rápida aplicação, por isso pesquisadores do Grupo de Estudos em Qualidade de Vida da Universidade Federal do Rio Grande do Sul desenvolveram para a Organização Mundial de Saúde a versão abreviada do WHOQOL-100, o WHOQOL-bref, que consta de 26 questões divididas em quatro domínios: físico, psicológico, relações sociais e meio ambiente.

Alguns anos depois, em 2003, o Grupo WHOQOL demonstrou interesse em desenvolver um instrumento para a avaliação da qualidade de vida em adultos idosos, partindo do pressuposto de que há especificidades referentes à fase do envelhecimento ${ }^{12}$.

O WHOQOL-100, nesta pesquisa, foi aplicado a 60 pessoas adultas que estavam responsáveis pelos cuidados dos pacientes internados nas Unidades de Urgência e Emergência do Hospital de Clínicas da Universidade Federal do Paraná: Pronto-Atendimento de Emergência, Pronto-Atendimento Adulto, Centro de Terapia Semi-Intensiva e Centro de Terapia Intensiva. Todos os participantes responderam o questionário após a 
assinatura do Termo de Consentimento Livre e Esclarecido.

Por ser um instrumento de autoavaliação, o WHOQOL-100 é autoexplicativo. Portanto, quando o participante não entendia o significado de alguma pergunta, o entrevistador relia a pergunta de forma lenta - não utilizava sinônimos ou explicações em outras palavras (aplicação assistida). Quando o respondente não podia ler em função de suas condições de saúde ou de alfabetização, o questionário era lido pelo entrevistador.

Os participantes foram escolhidos por sorteio: foi impressa a lista de pacientes de cada unidade, e cada um deles foi numerado. Em seguida, um profissional que estava na unidade no momento do sorteio, sem ter acesso à lista, escolheu dois números. Foi realizado um contato telefônico com os pacientes correspondentes a esses números e solicitado que o cuidador primário comparecesse ao hospital quando tivesse disponibilidade, normalmente em horário próximo ao da visita ao paciente.

Os resultados obtidos no estudo foram expressos por médias, medianas, valores mínimos, valores máximos e desvios padrão (variáveis quantitativas) ou por frequências e percentuais (variáveis qualitativas). Para avaliação da correlação entre a idade e os escores do WHOQOL, foi estimado o coeficiente de correlação de Spearman. Para comparação de dois grupos em relação ao escore do WHOQOL, foi considerado o teste não paramétrico de Mann-Whitney.

Essa comparação, para mais de dois grupos, foi feita usando-se o teste não paramétrico de Kruskal-Wallis. Valores de $\mathrm{p}<0,05$ indicaram significância estatística. Os dados foram analisados com o programa computacional Statistica v.8.0. A conversão das questões foi feita com o objetivo de padronizar todas as respostas do instrumento, de forma que a resposta mais positiva fosse 5. Por conseguinte, a resposta mais negativa seria 1. Assim, todas as questões de cada faceta foram convertidas para uma mesma escala, na qual o aumento gradativo da resposta equivale, na mesma proporção, ao aumento positivo do resultado da faceta. Além disso, em alguns outros domínios e associações, foi feita uma análise estatística descritiva dos dados.

Todos os dados foram coletados entre maio e outubro de 2011, sem identificação do participante pelo nome, mas por meio de um registro de inclusão dos pacientes que mostrava códigos, nomes e endereços para uso do pesquisador. Igualmente, os formulários do Termo de Consentimento assinados pelos pacientes foram mantidos em confidência restrita, em arquivo único. Esta pesquisa obedeceu à Resolução no $196 / 96^{13}$ da Comissão Nacional de Ética em Pesquisa, sendo aprovada pelo Comitê de Ética em Pesquisa em Seres Humanos do Hospital de Clínicas da Universidade Federal do Paraná.

\section{Resultados}

Entre os 60 participantes, predominou o sexo feminino ( $\mathrm{n}=47)$. A idade média foi de 43,2 anos, com prevalência da faixa etária de 31 a 50 anos (n =28), o que representa 46,7\% do total. Em seus estudos, os autores Perlini e Faro ${ }^{14}$ e Amendola et al. ${ }^{15}$ relataram que a mulher ainda é a principal responsável pelos cuidados com os membros da família, por motivos históricos e culturais. $\mathrm{Na}$ presente pesquisa, as participantes são filhas (30\%), esposas (16\%) ou mães (13,3\%). Quanto às unidades, os participantes ficaram assim distribuídos: Pronto-Atendimento de Emergência Adulto [PA Emergência] - 37\%; Centro de Terapia Semi-Intensiva [CTSI] - 28\%; Pronto-Atendimento de Observação Adulto [PA Observação] - 20\%; Centro de Terapia Intensiva [CTI] - 15\%.

As unidades de urgência e emergência foram criadas com o objetivo de prestar atendimento, diagnóstico e tratamento aos pacientes acidentados ou com uma emergência clínica, com ou sem risco iminente de vida.

Os pacientes internados nessas unidades geralmente estão em estado grave, com possibilidades de recuperação, exigindo assistência médica e de enfermagem permanente, além da utilização eventual de equipamento especializado ${ }^{16}$.

Os pacientes cujos cuidadores participaram deste estudo foram diagnosticados com doenças que não têm fator externo como causa e, durante o período de realização da pesquisa, estavam internados para tratamento de doenças infecciosas graves $(\mathrm{n}=28)$, infarto agudo do miocárdio $(\mathrm{n}=8)$, embolias pulmonares $(\mathrm{n}=12)$, acidente vascular cerebral $(\mathrm{n}=4)$, hipertensão arterial (n $=2$ ), insuficiência de orgãos de origem não traumática $(n=6)$.

Quando questionadas sobre o fato de exercerem alguma atividade laboral, 35 pessoas $(58,3 \%$ dos participantes) responderam não estar trabalhando no momento da entrevista. Quanto à escolaridade, aproximadamente $31 \%$ possuem Ensino Médio completo, enquanto 28\% possuem Ensino Fundamental incompleto, mas o grau de escolaridade variou de analfabeto (3\%) a Ensino Superior Completo (8\%). 
A qualidade de vida geral, composta pelas facetas satisfação com a vida, satisfação com a saúde, satisfação com a própria qualidade de vida e avaliação da qualidade de vida, obteve escore médio de 13,8, que traduz ausência de impacto negativo das facetas avaliadas na qualidade de vida. Os escores encontrados para os domínios evidenciaram médias maiores para nível de independência, aspectos espirituais/religião/crenças pessoais e relações sociais. As médias menores foram nos domínios físico, psicológico e ambiente. A variação obtida, de 12,5 e 17,0, mostra que esses valores estão acima da faixa de neutralidade, com tendência à valoração positiva, traduzindo qualidade de vida satisfatória, ou seja, pouco impacto negativo dos domínios na qualidade de vida (Figura 1).

\section{Associação de fatores com o resultado do WHOQOL}

\section{Idade $x$ WHOQOL}

Para cada um dos domínios do WHOQOL-100 e o escore geral, estimou-se o coeficiente de correlação de Spearman do escore com a idade. Testou-se a hipótese nula de que o coeficiente de correlação é igual a zero (ausência de associação) versus a hipótese alternativa de que o coeficiente é diferente de zero.

Na tabela abaixo são apresentados os coeficientes de correlação e os valores de p. Valores

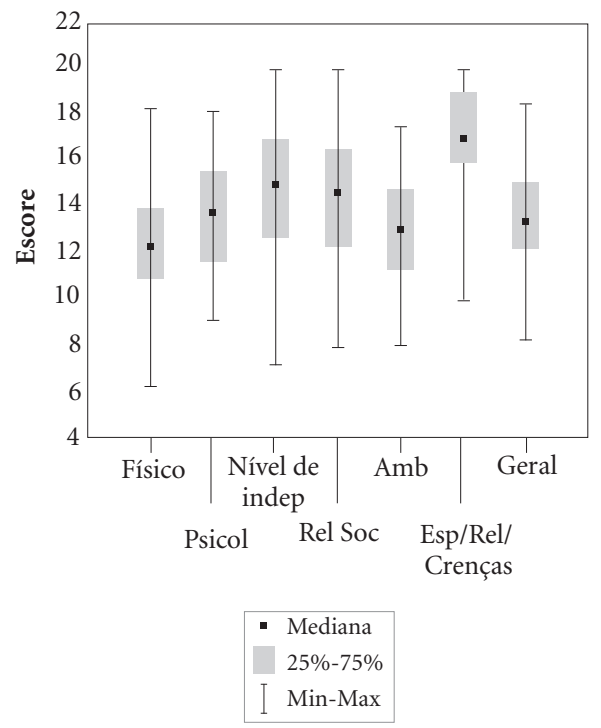

Figura 1. Resultados dos escores de cada domínio do WHOQOL-100. positivos do coeficiente de correlação indicam associação direta entre a idade e o escore, ou seja, valores altos (baixos) da idade correspondem a valores altos (baixos) do escore. Valores negativos do coeficiente de correlação indicam associação inversa entre a idade e o escore, ou seja, valores altos (baixos) da idade correspondem a valores baixos (altos) do escore.

Ainda em relação à idade, foram definidas três faixas: abaixo de 30 anos, de 31 a 50 anos e acima de 50 anos. Com isso, para cada domínio e para o escore geral, testou-se a hipótese nula de que o escore é igual nas 3 faixas etárias versus a hipótese alternativa de que pelo menos uma faixa etária tem resultado do escore diferente das demais. Na tabela abaixo são apresentados os coeficientes de correlação e os valores de p. Percebe-se uma diferença significativa entre idade e os domínios físico e nível de independência (Tabela 1).

Esses indicadores são interessantes e levam à reflexão sobre a possibilidade de os cuidados desencadearem situações de vulnerabilidade nas dimensões física, psíquica e espiritual, interferindo diretamente na qualidade de vida dos cuidadores. Com relação à diferença significativa na associação entre a idade e o nível de independência, pode-se pensar que, em situações de internação e adoecimento de algum membro da família, as pessoas responsáveis por acompanhar e cuidar do paciente internado sentem-se dependentes da ajuda de outros. Essa ajuda pode ser financeira ou com o revezamento entre os membros familiares. A diminuição do nível de independência pode ser justificado pelo fato de que $58 \%$ dos participantes não estavam trabalhando na época da pesquisa.

Tabela 1. Relação entre os coeficientes de correlação de Spearman e os valores de p, com relação à idade.

\begin{tabular}{|c|c|c|c|}
\hline & $\mathbf{N}$ & $\begin{array}{c}\text { Coef. de } \\
\text { correlação } \\
\text { de Spearman }\end{array}$ & $\begin{array}{l}\text { Valor } \\
\text { de p }\end{array}$ \\
\hline Idade $\mathrm{x}$ físico & 60 & $-0,30$ & 0,018 \\
\hline Idade x psicológico & 60 & $-0,15$ & 0,259 \\
\hline $\begin{array}{l}\text { Idade x nível de } \\
\text { independência }\end{array}$ & 60 & $-0,32$ & 0,013 \\
\hline Idade $\mathrm{x}$ relações sociais & 60 & 0,00 & 0,976 \\
\hline Idade $\mathrm{x}$ ambiente & 60 & $-0,09$ & 0,515 \\
\hline $\begin{array}{l}\text { Idade } \mathrm{x} \text { aspectos espirituais/ } \\
\text { religião/crenças pessoais }\end{array}$ & 60 & 0,11 & 0,391 \\
\hline Idade $x$ GERAL & 60 & $-0,18$ & 0,170 \\
\hline
\end{tabular}


Com relação à idade, os resultados apresentam uma diferença significativa com relação à idade e o domínio 'crenças pessoais' $(\mathrm{p}=0,014)$.

\section{Gênero x WHOQOL}

Para cada um dos domínios do WHOQOL e o escore geral, testou-se a hipótese nula de que o resultado do escore é igual para os gêneros masculino e feminino, versus a hipótese alternativa de resultados diferentes. $\mathrm{Na}$ tabela abaixo são apresentadas estatísticas descritivas dos escores de acordo com os gêneros e os valores de $\mathrm{p}$ dos testes estatísticos $(\mathrm{p}<0,05)$ (Tabela 2$)$.

\section{Discussão}

Sabe-se que o homem é um ser de relações e a família, um ambiente psicodinâmico significativo em nossa condição humana. O sistema familiar se transforma diante de mudanças e sofre os efeitos das ações dos membros que o compõem, exigindo adaptações que determinam as características desse sistema ${ }^{17}$. Quanto mais flexibilidade a família apresentar diante de uma nova situação de vida, mais significativo se tornará o sistema, exercendo uma de suas funções - o apoio e a proteção psicossocial entre seus membros -, o que inclui o próprio ato de cuidar.

Os problemas de saúde são um dos eventos estressantes que mais desafiam as famílias, pois requerem recursos e esforços para enfrentamento e adaptação, bem como contribuições para o bom funcionamento do sistema familiar no momento em que um dos membros adoece. De acordo com Walsh ${ }^{18}$, a família precisa criar um significado para o sofrimento, a fim de preservar a sensação de equilíbrio, reorganizar-se diante da crise e ser flexível para atender as necessidades de todos os membros, sendo uma delas a prestação de cuidado ao membro que adoece.

Segundo Azevedo e Santos ${ }^{19}$, a palavra 'cuidado' tem origem no latim cura ou coera e era usada quando se demonstrava uma atitude de preocupação, atenção e interesse, geralmente nas

Tabela 2. Descrição dos escores de gênero e o domínio psicológico.

\section{Psicológico}

$\begin{array}{lllllll}\text { Feminino } & 47 & 13,4 & 13,4 & 9,2 & 18,2 & 2,4\end{array}$

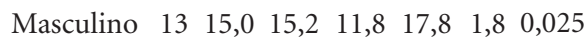

relações de afeto. O cuidado surge a partir da necessidade de uma pessoa ser atendida por outra, o cuidador. $\mathrm{O}$ autor ainda afirma que, além das ações de bom trato, o ato de cuidar significa preocupação, visto que está intimamente ligado ao outro, sinal de que a palavra é reveladora de uma vivência ambivalente.

O impacto subjetivo da doença e as tarefas desenvolvidas pelo cuidador, ao longo do tempo e associadas a diversos fatores, acabam constituindo eventos estressores significativos, trazendo consequências e demandas emocionais para o cuidador $^{20}$.

Para Walsh ${ }^{18}$, o cuidado prolongado e a falta de manejo dos profissionais de saúde causam nos membros que cuidam a sensação de desamparo, confusão e frustração. O autor afirma que aproximadamente $80 \%$ dos cuidadores prestam ajuda 7 dias por semana, 4 horas por dia. Suas necessidades devem ser atendidas e respeitadas, entendendo-se como as pessoas atingidas e os membros da sua rede social percebem, vivem e superam os desafios que um adoecimento e uma internação provocam.

O papel do cuidador é fundamental na assistência ao doente e na sua manutenção na comunidade. No entanto, tal papel pode interferir diretamente na convivência pessoal, familiar e social do sujeito que cuida, trazendo transformações no seu dia a dia, como também em sua qualidade de vida, o que faz com que avaliações nesse sentido se tornem importantes ${ }^{21}$.

A questão é importante, pois os hábitos culturais podem ser negociados nas relações sociais e aceitos pela sociedade, construindo a forma como a pessoa compreende o mundo e o contexto em que está inserida, em especial a família, o primeiro meio de interação social, no qual se encontra a primeira instância cuidadora dos seus membros ${ }^{22}$.

De acordo com $\mathrm{McDaniel}^{23}$, a família, muitas vezes, funciona como recurso para um momento estressante e de sofrimento, como o adoecimento e a internação, trazendo ações de colaboração e apoio mútuo. Por isso é preciso incluir na comunicação e na intervenção dos profissionais envolvidos o respeito às culturas familiares.

Para a presente pesquisa, foram encontrados 89 artigos com as palavras-chaves "qualidade de vida" e "cuidador". Em contrapartida, foram localizados 1.086 artigos sobre "qualidade de vida do paciente" na base de dados LILACS, entre os anos 2000 e 2011. Há um aumento significativo a partir de 2004, sendo brasileiros aproximadamente $94 \%$ dos estudos. Esses dados mostram 
que existem 12 vezes mais trabalhos sobre qualidade de vida do paciente em comparação à qualidade de vida do cuidador.

Quanto à qualidade de vida, deve-se considerar que ela é resultante social da construção coletiva dos padrões de conforto e bem-estar que determinada sociedade estabelece, como parâmetros, para si ${ }^{24}$. No campo da saúde, o discurso da relação entre saúde e qualidade de vida tem como principal estratégia e foco a promoção da saúde.

Nas discussões sobre o tema, a Organização Mundial da Saúde (OMS) tem tido papel relevante no desenvolvimento do conceito e da sua operacionalização, com forte tendência a considerar a multidimensionalidade e a subjetividade como dois fenômenos fundamentais no estudo da qualidade de vida. Por conta desse consenso, ponto de partida para a construção do instrumento para avaliar a qualidade de vida, a $\mathrm{OMS}^{3}$ definiu qualidade de vida como "a percepção do indivíduo de sua posição na vida no contexto da cultura e sistemas de valores nos quais ele vive e em relação aos seus objetivos, expectativas e preocupações".

Na década de noventa, ocorreu uma proliferação de instrumentos para avaliar a qualidade de vida, a maioria desenvolvida nos Estados Unidos. Cresceu também, nesse período, o interesse pela tradução desses instrumentos para aplicação em outros países ${ }^{25}$.

A percepção do impacto subjetivo da doença depende principalmente da forma como o cuidador avalia sua condição. A preocupação com o conceito de qualidade de vida refere-se a um movimento dentro das ciências humanas e biológicas no sentido de valorizar parâmetros mais amplos que o controle de sintomas, a diminuição da mortalidade ou o aumento da expectativa de $\operatorname{vida}^{26-28}$.

\section{Análise estatístico-descritiva dos dados}

À questão "Você consegue dos outros o apoio que necessita?", 32 pessoas (53,3\% dos entrevistados) responderam "muito" ou "completamente". Ainda sobre esse tema, aproximadamente $73 \%$ dos entrevistados relataram dividir e/ou revezar os cuidados com a pessoa internada. Quando alguém adoece, experimenta, junto com a doença, sentimentos como ansiedade, confusão e depressão. Por isso o sucesso da cura e a superação de um evento significativo como o adoecer depende da mobilização dos recursos familiares e comunitários, por meio de atos de colaboração. Mesmo com todos os desafios implícitos no ato de cuidar, a família deve se manter ao lado do paciente, sendo indispensável ao longo do tratamento ${ }^{18}$.

$\mathrm{O}$ resultado do escore tem uma diferença estatisticamente significativa para indivíduos com grau de parentesco pai/mãe/filho/cônjuge e indivíduos com outro grau de parentesco (13,5 X $15 ; p=0,032$ ). Isso significa que indivíduos com grau de parentesco familiar têm escores menores relacionados à qualidade de vida quando se veem envolvidos com o ato de cuidar. No decorrer do ciclo de vida, a doença atinge todos os indivíduos e, consequentemente, aqueles que estão mais próximos, normalmente seus familiares, trazendo sofrimento e acarretando prestação de cuidados.

A história do relacionamento entre o paciente e o cuidador, bem como os recursos pessoais e relacionais de enfrentamento, devem ser considerados. A inclusão da família favorece a criação de um significado para suportar a reorganização da crise a curto prazo e desenvolver flexibilidade familiar durante a internação, assim como um encorajamento para o bom funcionamento e o bem-estar relacional ${ }^{18}$.

Os itens F24.1, F24.2, F24.3 e F24.4 do WHOQOL-100 referem-se aos temas religiosidade e espiritualidade. Tais práticas, consideradas importantes aliados das pessoas que sofrem e/ou estão doentes, agora começam a ser valorizadas por estudos na área de saúde. Segundo Panzini et al. ${ }^{29}$, essas questões pretendem contribuir para o aprofundamento das dimensões religiosa e espiritual ligadas ao fenômeno saúde-doença, visto que, na medicina oriental, essa integração já vem sendo realizada há mais tempo ${ }^{30}$. Para Andrade et al. ${ }^{2}$, a religiosidade também é uma forma de enfrentar determinado sofrimento, fazendo com que a fé e as crenças pessoais auxiliem na perspectiva de melhora do paciente e no despertar para uma nova vida. Aproximadamente $90 \%$ dos entrevistados responderam "bastante" ou "extremamente" quando questionados se as crenças pessoais ajudam a enfrentar e entender as dificuldades da vida - como, por exemplo, o adoecimento de um membro da família e o ato de cuidar - e se permitem que os participantes encontrem sentido para o sofrimento nessa fase de sua vida.

As crenças espirituais auxiliam na compreensão do desafio de ser cuidador, constituindo um recurso a mais de enfrentamento ${ }^{18}$, enquanto as crenças pessoais desempenham um papel de consolo e confiança de que dias melhores virão, inspirando coragem e fazendo emergirem soluções para a resolução de problemas e conflitos. 


\section{Considerações Finais}

Tais achados estatísticos podem reforçar a importância do desenvolvimento de ações na linha de cuidado para a equipe multidisciplinar atender esses cuidadores no ato de cuidar, durante a internação do paciente, e propor ações educativas de orientação para cuidadores e familiares, para que eles possam continuar a desempenhar as atividades mesmo quando estiverem longe do ambiente hospitalar.

Percebeu-se, na presente pesquisa, a existência de uma rede de acolhimento quando os participantes disseram poder contar com outras pessoas para revezar nos cuidados durante a internação e após a alta hospitalar. O teste mostrou que os participantes recebiam dessa rede apoio financeiro e ajuda para reorganizar a rotina familiar, de modo a prevenir que um dos cuidadores se sobrecarregasse com a atenção prestada durante o tratamento.

Pretende-se, com a divulgação dos resultados deste estudo, contribuir com um referencial para a equipe multiprofissional de saúde no planejamento de ações de colaboração direcionadas às famílias de pessoas internadas devido a uma doença crônica, como estratégia para o enfrentamento do sofrimento e da angústia, evitando que o impacto de uma internação traga consequências prejudiciais à qualidade de vida do cuidador familiar.

Acredita-se que, com familiares melhor preparados para realizar as ações de cuidado após a alta hospitalar, as chances de ocorrerem reinternações ou óbitos serão menores, fazendo com que tanto paciente quanto cuidadores se sintam acolhidos e amparados por uma linha de cuidado institucional, profissional - que vai desde a atenção primária, promoção da saúde e prevenção (realizada geralmente nas unidades de saúde) até a assistência em níveis mais graves, como a prestada em instituições hospitalares de referência no atendimento em alta complexidade.

A ausência de programas de apoio ao cuidador familiar, que visam acolher as novas demandas que as doenças crônicas degenerativas acarretam às famílias, aliada à falta de recursos financeiros e de apoio da comunidade, acaba agravando o problema na instância da saúde pública. Nessa perspectiva, salienta-se a necessidade da criação de políticas públicas, recursos e ações de colaboração para a população pouco assistida, considerando-se todas as especificidades psíquicas, culturais e sociais aqui apresentadas.

Sabe-se que, para viabilizar essas ações, é preciso que todos os profissionais de saúde estejam abertos a compreender o sistema familiar e a conhecer os aspectos relacionados à história de vida e do adoecimento, bem como a trajetória do enfrentamento percorrida pelos cuidadores antes, durante e após a internação em ambiente hospitalar. Os resultados da pesquisa sugerem que os cuidadores são parte fundamental no processo de tratamento e recuperação, daí a necessidade de planejar ações de colaboração, de suporte social, psicológico e espiritual nesse momento de vulnerabilidade psíquica do cuidador, para então promover qualidade de vida satisfatória para todos os envolvidos no processo saúde-doença.

\section{Colaboradores}

TM Dallalana e MGR Batista participaram igualmente de todas as etapas de elaboração do artigo. 


\section{Referências}

1. Martins JJ, Schneider DG, Coelho FL, Nascimento ER, Albuquerque GL, Erdmann AL, Gama FO. Avaliação da qualidade de vida de idosos que recebem cuidados domiciliares. Acta Paul. Enferm. 2009; 22(3):265-271.

2. Andrade, LM, Costa MF, Caetano JA, Soares E, Beserra EP. A problemática do cuidador familiar do portador de acidente vascular cerebral. Rev. Esc. Enferm. USP 2009; 43(1):37-43.

3. Organização Mundial da Saúde (WHO). WHOQOL: mesuring quality of life. Geneva: WHO; 1997.

4. Morin E. O paradigma perdido. $5^{\mathrm{a}}$ ed. Portugal, Ed. Biblioteca Universitária; 1973.

5. Maturana H. Cognição, ciência e vida cotidiana. Belo Horizonte: Ed. UFMG; 2001.

6. Bateson G. Espíritu y naturaleza. Buenos Aires: Ed. Amorrortu; 1993.

7. Bronfenbrenner U. A ecologia do desenvolvimento humano. Porto Alegre: Artes Médicas; 1994.

8. Minayo MCS. O desafio do conhecimento. São Paulo, Rio de Janeiro: Hucitec, Abrasco; 1992.

9. Spink MJP. Práticas discursivas e produção de sentido no contidiano: Aproximações teóricas e metodológicas. $3^{\mathrm{a}} \mathrm{ed}$. São Paulo: Cortez; 2004.

10. Fleck MP, Leal OF, Louzada S, Xavier EC, Vieira G, Santos L, Pinzon V. Desenvolvimento da versão em português do instrumento de avaliação de qualidade de vida da OMS (WHOQOL-100). Rev. Bras. Psiquiatr. 1999; 21(1):19-28.

11. Fleck MPA, Louzada S, Xavier M, Chachamovich E, Vieira G, Santos L, Pinzon V. Aplicação da versão em português do instrumento abreviado de avaliação da qualidade de vida "WHOQOL-bref”. Rev Saude Publica 2000; 34(2):178-183.

12. Fleck MPA, Chachamovich E, Trentini CM. Projeto WHOQOL-OLD: método e resultados de grupos focais no Brasil. Rev Saude Publica 2003; 37(6):793-799.

13. Brasil. Ministério da Saúde (MS). Conselho Nacional de Saúde. Resolução no 196 de 10 de outubro de 1996. Diretrizes e Normas Regulamentadoras de Pesquisas Envolvendo Seres Humanos. Diário Oficial da União 1996; 16 out.

14. Perlini MNOG, Faro ACM. Cuidar de pessoa incapacitada por acidente vascular cerebral no domicílio: o fazer do cuidador familiar. Rev. Esc. Enferm. USP 2005; 39(2):154-163.

15. Amendola F, Oliveira MAC, Alvarenga MRM. Influência do apoio social na qualidade de vida do cuidador familiar de pessoas com dependência. Rev. Esc. Enferm. USP 2011; 45(4):884-889.

16. Brasil. Ministério da Saúde (MS). Conceitos e definições em saúde. [Internet].1977. [acessado 2012 jan 20]. Disponível em: http://bvsms.saude.gov.br/bvs/publicacoes /0117conceitos.pdf.

17. Minuchin S. Famílias: funcionamento e tratamento. Porto Alegre: Artes Médicas; 1982.
18. Walsh F. Fortalecendo a resiliência familiar. São Paulo: Roca; 2005.

19. Azevedo GR, Santos VLCG. Cuida-dor (d)eficiente: as representações sociais de familiares acerca do processo de cuidar. Rev. Latino-am. Enfermagem 2006; 14(5):762-769.

20. Silveira TM, Caldas CP, Carneiro TF. Cuidando de idosos altamente dependentes na comunidade: um estudo sobre cuidadores familiares principais. Cad Saude Publica 2006; 22(8):1629-1638.

21. Martins T, Ribeiro JP, Garret C. Estudo de validação do questionário de avaliação da sobrecarga para cuidadores informais. Psicol. saúde doença 2003; 4(1):131-148.

22. Leite SN, Vasconcellos MPC Negociando fronteiras entre culturas, doenças e tratamentos no cotidiano familiar. Hist. cienc. saúde-Manguinhos 2006; 13(1):91-112.

23. McDaniel SH. Terapia familiar médica: um enfoque biopsicossocial às famílias com problemas de saúde. Porto Alegre: Artes Médicas; 1994.

24. Minayo MCS, Hartz ZMA, Buss PM. Qualidade de vida e saúde: um debate necessário. Cien Saude Colet 2000; 5(1):4-5.

25. Bullinger M, Anderson R, Cella D. Developing and evaluating cross-cultural instruments from minimum requirements to optimal models. Qual. life res. 1993; 2(6):451-445.

26. Paulino FG, Duarte DT, Brito M, Degaki N, Schwartz D. Perfil do cuidador profissional de idosos com demência. In: $3^{\circ}$ Congresso Ibero-americano de Psicogerontologia; 2009, São Paulo, Brasil. São Paulo; PUCSP; 2099.

27. Fonseca NR, Penna AFG. Perfil do cuidador familiar do paciente com seqüela de acidente vascular encefálico. Cien Saude Colet 2008; 13(4):1175-1180.

28. Rebouças M, Galera SC, Pereira SRM, Pereira MG. Que informações são necessárias para o conhecimento da situação de saúde do idoso? Cien Saude Colet 2008; 13(4):1305-1312.

29. Panzini RG, Maganha C, Rocha NS, Bandeira DR, Flech M. Validação brasileira do Instrumento de Qualidade de Vida/espiritualidade, religião e crenças pessoais. Rev Saude Publica 2011; 45(1):153-165.

30. Moreira-Almeida A, Lotufo-Neto F, Koenig HG. Religiousness and mental health: A review. Rev. Bras. Psiquiatr 2006; 28(3):242-250.

Artigo apresentado em 09/08/2013

Aprovado em 31/08/2013

Versão final apresentada em 09/09/2013 